\title{
Direct Analysis of Aerosolized Chemical Warfare Simulants Captured on a Modified Glass-Based Substrate by ‘Paper-Spray' Ionization
}

\author{
Elizabeth S. Dhummakupt ${ }^{\dagger, \S}$, Phillip M. Mach ${ }^{\ddagger, \S}$, Daniel Carmany ${ }^{\ddagger}$, Paul S. Demond ${ }^{\ddagger}$, \\ Theodore S. Moran ${ }^{\dagger}$, Theresa Connell ${ }^{\ddagger}$, Harold S. Wylie", Nicholas E. Manicke ${ }^{\perp}, \mathrm{J}$. \\ Michael Nilles ${ }^{\ddagger, *}$, and Trevor Glaros ${ }^{\dagger, *}$ \\ † Research and Technology Directorate, US Army Edgewood Chemical Biological \\ Center (ECBC), Aberdeen Proving Ground, MD 21010, USA \\ ‡ Excet, Inc. 6225 Brandon Ave, Suite 360, Springfield, VA 22150, USA \\ "TriMech Services, LLC, 4461 Cox Rd \# 302, Glen Allen, VA 23060, USA \\ ${ }^{\perp}$ Department of Chemistry and Chemical Biology, Indiana University-Purdue University \\ Indianapolis, Indianapolis, IN 46202, USA \\ Author Information \\ Corresponding Authors \\ *T.G. $\quad$ trevor.g.glaros.civ@mail.mil \\ *J.M.N. mnilles@gmail.com \\ Author Contributions \\ $\S$ E.S.D. and P.M.M. contributed to this work equally, with joint authorship. \\ Notes \\ The authors declare no competing financial interest.
}

Distribution Statement A: Approved for public release; distribution is unlimited.

This is the author's manuscript of the article published in final edited form as:

Dhummakupt, E. S., Mach, P. M., Carmany, D., Demond, P. S., Moran, T. S., Connell, T., ... Glaros, T. (2017). Direct Analysis of Aerosolized Chemical Warfare Simulants Captured on a Modified Glass-Based Substrate by "Paper-Spray" Ionization. Analytical Chemistry, 89(20), 10866-10872. https://doi.org/10.1021/acs.analchem.7b02530 


\begin{abstract}
Paper spray ionization mass spectrometry (PS-MS) offers a rapid alternative platform requiring no sample preparation. Aerosolized CWA simulants, trimethyl phosphate, dimethyl methylphosphonate, and diisopropyl methylphosphonate, were captured by passing air through a glass fiber filter disk and simultaneously capturing it directly onto a disposable paper spray cartridge. CWA simulants were aerosolized at varying concentrations using an in-house built aerosol chamber. A custom 3D-printed holder was designed and built to facilitate the aerosol capture onto the paper spray cartridges. The air flow through each of the collection devices was maintained equally to assure the same volume of air sampled across methods. Each approach yielded linear calibration curves with $R^{2}$ values between 0.98-0.99 for each compound and similar limits of detection in terms of disbursed aerosol concentration. While the glass fiber filter disk has a higher capture efficiency $(\approx 40 \%)$, the paper spray method produces analogous results even with a lower capture efficiency $(\approx 1 \%)$. Improvements were made to include glass fiber filters as the substrate within the paper spray cartridge consumable. Glass fiber filters were then treated with ammonium sulfate to decrease chemical interaction with the simulants. This allowed for improved direct aerosol capture efficiency $(>40 \%)$. Ultimately, the limits of detection were reduced to levels comparable to current worker population limits of $1 \times 10^{-6} \mathrm{mg} / \mathrm{m}^{3}$.
\end{abstract}

Distribution Statement A: Approved for public release; distribution is unlimited. 
Despite nearly a century of efforts ${ }^{1}$ to ban the development, production, and stockpiling of CWAs, their threat to warfighters, first responders, and even civilian populations remains. There have been multiple legislative and international documents which collectively aim to enhance regulation and ultimately lead to the destruction of these objectionable chemicals ${ }^{2}$. CWAs have been deployed recently in wartime theaters, such as the Ghouta chemical attack in $2013^{3}$, which was the deadliest use of CWAs since the Iran-Iraq War, and more recently, the Khan Shaykhun attack of $2017^{4}$. Presently, there is a myriad of detection methods in battlefield and forensic situations, such as ion mobility spectrometry $(\mathrm{IMS})^{5-9}$, colorimetric paper, ${ }^{6}$, and mass spectrometry (MS) ${ }^{10-11}$. Unfortunately, many of these technologies are less effective at directly detecting compounds in aerosolized form, such as from an explosively disbursed chemical munition.

At present, most fielded systems for the detection of aerosolized CWAs contain an IMS based sensor. While IMS has the advantages of simplicity of components, sensitivity to low ppm levels ${ }^{7}$, portability, rapid analysis times, and low $\operatorname{cost}^{12}$, the platform has a number of disadvantages, including inability to directly analyze aerosols, sensitivity to temperature and humidity, causing ion clustering (similar to aerosols), false alarms caused by interferences due to poor resolution, and detector saturation from exposure to high concentrations which leads to long clearance times between runs ${ }^{7,12}$. Most field IMS devices cannot identify CWAs at acceptable exposure levels (AEL) of $0.0001 \mathrm{mg} / \mathrm{m}^{3}$ for nerve agents and $0.003 \mathrm{mg} / \mathrm{m}^{3}$ for blister agents ${ }^{13-15}$.

M9 colorimetric paper is also used in the field for CWA detection ${ }^{6,9}$. It has the advantage of being inexpensive and simple to use with a fast response rate $(\approx 30$ seconds). The paper is selective in that only the particular reagent reacts with the specific class of CWA (e.g., "V", "G", "H" agent) ${ }^{6,12}$. Because of this specificity, multiple different paper detectors are needed. Additionally, the analysis of these papers rely on human visual processing or optical sensors, which can be affected by differences in ambient light, color perception, or color blindness ${ }^{12}$ and only give qualitative results ${ }^{9}$. The M9 paper is also reactive to vapors from insecticides, smoke, acetone, gasoline, and bleach solutions causing a high false positive rate ${ }^{16}$.

Membrane inlet mass spectrometry (MIMS) is continually being developed as a technique for security and forensic applications such as CWAs ${ }^{10}$ and illicit drug synthesis detection ${ }^{10-11}$. It has the advantages of minimal sample preparation, sensitivity in the parts per billion $(\mathrm{ppb})^{17}$ to parts per trillion $(\mathrm{ppt})^{18}$ levels and short response times $^{18-19}$. However, MIMS has difficulty with polar molecules and larger (>500 Da) molecules $^{18}$. Moreover, like IMS, aerosols need to be completely vaporized to pass through the membrane natively. Additionally, high-concentration aerosols would take a long time to clear out of the system for subsequent detections. There have been further efforts to create specialized instrumentation to measure aerosol chemical composition ${ }^{20-}$ 26. Mass analysis techniques are presently less effective at detection of aerosolized CWAs, as contrasted to vapor phase detections. Thus, newly developed analysis techniques must be robust enough to provide improved aerosol results.

Distribution Statement A: Approved for public release; distribution is unlimited. 
Paper spray (PS) and associated substrate spray are an ambient ionization techniques that allow for direct sampling with little to no sample preparation and rapid MS analysis ${ }^{27-29}$. Samples are collected or deposited directly onto the paper substrate from biological and environmental sources and analyzed by MS without the need for desorption/extraction ${ }^{30-32}$. Currently, PS-MS has been reported to analyze CWA simulants and CWA hydrolysis products $^{33}$ in biological matrices, as well as food and environmental samples containing pesticides and herbicides ${ }^{32,34}$, which have chemical similarities to CWAs. In this study, PS-MS analysis of aerosolized CWA simulants of Gseries nerve agents (e.g., sarin, soman), which are useful for defensive research ${ }^{35-36}$, were explored in both laboratory and field (real-world) settings. The capture approach discussed here further demonstrates paper spray's potential as an MS ionization technique for chemical threats, as it is also compatible with CWA-contaminated clinical ${ }^{33}$ and environmental samples.

\section{Experimental Section}

\section{Chemicals and Materials}

Optima-grade high-performance liquid chromatography (HPLC) solutions of methanol, acetonitrile (ACN), and water were purchased from Thermo Fisher Scientific (Waltman, MA, USA), in addition to the $25 \mathrm{~mm}$ borosilicate glass fiber A/E filters (Pall Corporation, Port Washington, NY, USA). HPLC grade formic acid and ammonium sulfate were purchased from Sigma Aldrich (St. Louis, MO, USA). Trimethyl phosphate (TMP), diisopropyl methylphosphonate (DIMP), and dimethyl methylphosphonate (DMMP) were also purchased from Sigma Aldrich. The $d_{9} T M P$ was purchased from Cerilliant (Round Rock, TX, USA). The ${ }^{13} \mathrm{Cd}_{3}$ DIMP was synthesized by Dr. Bob Williams and Mark Alverez at the Los Alamos National Laboratory (Los Alamos, NM, USA) and used as the internal standard for both TMP and DMMP. Isotope label positions have been described previously ${ }^{33}$. Paper spray cartridges containing ET31 chromatography paper (Whatman plc, Maidstone, U.K.) were purchased from Prosolia (Indianapolis, IN, USA).

\section{Sample Preparation: Aerosolization}

The CWA simulants were diluted from neat material to a stock concentration of 1 $\mathrm{mg} / \mathrm{mL}$ in a solvent appropriate for solubility (high purity methanol for DIMP and HPLC grade water for all others). From the $\approx 1 \mathrm{mg} / \mathrm{mL}$ stock solutions described above, solutions of the CWA simulants were made at concentrations of $3,30,60,90,120$, and $180 \mu \mathrm{g} / \mathrm{mL}$ in methanol for testing in an aerosol chamber. This procedure was repeated for the lower concentrations of $2,5,25,50,100,250$, and $500 \mathrm{ng} / \mathrm{mL}$.

Substrate Preparation: Glass Treatment

Distribution Statement A: Approved for public release; distribution is unlimited. 
The glass fiber filters were treated with ammonium sulfate, as previously described $^{37}$. Briefly, a $500 \mathrm{mM}$ ammonium sulfate solution was prepared in water. Approximately $200 \mu \mathrm{L}$ of solution was applied to the borosilicate glass fiber disk, and baked at $37^{\circ} \mathrm{C}$ for 1 hour. Each disk was then washed with $500 \mu \mathrm{L}$ of water and blotted dry. A drying process at $60^{\circ} \mathrm{C}$ for 5 minutes, or until completely dry, was performed. The washing/drying process was then repeated.

\section{Aerosol Chamber Design and Conditions}

Paper spray cartridge collections were conducted while directly connected to the outer plenum of a $50 \mathrm{cc}$ inner volume modular exposure chamber $(\mathrm{CH}$ Technologies, Westwood, NJ, USA). The chamber had a flood rate of 20 liters per minute and was maintained under static negative pressure of -0.036 psig. Flow was maintained using a Teledyne Hastings Instrument LS1D Laminar Flow Element (Hampton, VA, USA), and pressure was maintained with Brooks Instruments mass flow controllers, model 5751E and readout and controller electronics model 0154 (Hatfield, PA, USA) connected to house vacuum.

Aerosol was generated using the aerosolization solutions (described above) through a double needle atomizer ${ }^{38}$. The solutions were introduced into the system by a Harvard Apparatus Syringe Drive, model 55-222 (South Natick, MA, USA) using a Hamilton 1000 series removable needle $1.0 \mathrm{~mL}$ syringe, model 1750-1000 $\mu \mathrm{L}$. Liquid solution was driven at a fixed flow rate of $10 \mu \mathrm{L} / \mathrm{min}$. The outer needle flowed ultra-zero grade compressed air at 60 psig to achieve liquid drop sheering causing aerosolization of the solution. This process occurred in a $2 \mathrm{~L}$ stainless steel custom drying chamber which was connected to the exposure chamber using stainless steel tubing.

Before exposure, a 2-3 minute priming period was used to allow chamber concentrations to equilibrate. Identical 3D printed cartridge samplers and glass fiber filter holders were docked to the chamber at the appropriate ports and new Prosolia cartridges and glass fiber filters were used for each run. Exposures lasted 10 minutes (Generation One) or 2 minutes (Generation Two), after which all collected samples were removed and prepared for analysis.

\section{Paper Spray Cartridge Holder Design and Integration}

The paper spray cartridge holders were designed using Solidworks 2014 and 2016 (Waltham, MA, USA). The Generation One and Two design was 3D-printed on 3D Systems Vanguard HS (Rock Hill, SC, USA) using selective laser sintering (SLS) with glass-filled nylon materials. Excess powder from the build process was removed using an air nozzle. Generation Two was fabricated using an Objet500 Connex (Eden Prairie, MN), Veroclear RGD810 thermoplastic material. All parts were cleaned and rinsed in an ultrasonic bath with Liqui-Nox from Alconox (White Plains, NY, USA) detergent. Capture cartridge holders were connected to the aerosol chamber using a 4 inch length of UltraChemical-Resistant Versilon PVC Tubing (McMaster-Carr, Elmhurst, IL). Pumping was

Distribution Statement A: Approved for public release; distribution is unlimited. 
provided by T2-03 (2.5 L/min max flow; Generation One) and TS002 (6.0 L/min max flow; Generation Two) diaphragm pumps from Parker (Hollis, NH, USA).

\section{Sample Extraction and Triple Quadrupole (QqQ) Mass Spectrometer Data Acquisition}

The analyte was extracted from the glass fiber filtration disk with $1 \mathrm{~mL}$ of methanol containing the internal standards ${ }^{13} \mathrm{Cd}_{3}$ DIMP and $\mathrm{d}_{9}$ TMP for 1 hour. All samples were analyzed on an Agilent Technologies 1290 LC (Santa Clara, CA, USA) with an Agilent Technologies 6490 Triple Quad Mass Spectrometer (Santa Clara, CA, USA). The samples were separated by liquid chromatography using a 4.6 × $50 \mathrm{~mm}$ Agilent Technologies C18 column with $1.8 \mu \mathrm{m}$ particle size. The mobile phase used was $100 \%$ ACN with $0.1 \%$ formic acid and the aqueous phase was $100 \% \mathrm{H}_{2} \mathrm{O}$ with $0.01 \%$ formic acid. The flow was $1.0 \mathrm{~mL} / \mathrm{min}$ with an injection volume of $1 \mu \mathrm{L}$. The gradient was $5 \% / 95 \%\left(\mathrm{ACN} / \mathrm{H}_{2} \mathrm{O}\right)$ from 0.2 to 3.2 minutes, ramp to $90 \% / 10 \%$ from 3.2 to 3.4 minutes, hold from 3.4 to 3.6 minutes, and change to $5 \% / 95 \%$ from 3.6 to 5.0 minutes. The mass spectrometer was operated in multiple reaction monitoring (MRM) mode using electrospray ionization in positive ion mode. 3500 VDC was applied across the capillary. The nitrogen drying gas flow was $15 \mathrm{~L} / \mathrm{min}$ controlled at $150^{\circ} \mathrm{C}$ and the sheath gas flow was $11 \mathrm{~L} / \mathrm{min}$ and maintained at $250^{\circ} \mathrm{C}$. The precursors and transitions for the five simulant compounds were as follows: TMP $(\mathrm{m} / \mathrm{z} 141.0 \rightarrow 127.0)$ at collision energy (CE) $16 \mathrm{~V}, \mathrm{~d}_{9} \mathrm{TMP}(\mathrm{m} / \mathrm{z} 150.1 \rightarrow 133.1)$ at CE $16 \mathrm{~V}$, DMMP $(\mathrm{m} / \mathrm{z} 125.0 \rightarrow 111.0)$ at CE $12 \mathrm{~V}$, $\operatorname{DIMP}(\mathrm{m} / \mathrm{z} 203.1 \rightarrow 161.0)$ at CE $4 \mathrm{~V}$, and ${ }^{13} \mathrm{Cd}_{3} \operatorname{DIMP}(\mathrm{m} / \mathrm{z} 207.1 \rightarrow 165.1)$ at CE $4 \mathrm{~V}$

\section{Paper Spray Ionization \& Orbitrap Mass Spectrometer Data Acquisition}

The Velox360 autosampler and ionization source from Prosolia was used in this study. For the calibration curve, a $10 \mu \mathrm{L}$ aliquot of mixed CWA simulant and internal standard was pipetted onto the PS cartridge. For aerosol samples on PS cartridges, a $10 \mu \mathrm{L}$ aliquot of the internal standard mixture was added after aerosol collection and prior to MS analysis. The internal standard concentration was fixed across all analytes prepared regardless of analyte concentration. During the solvent addition step, pump A was programmed to dispense $3 \mu \mathrm{L}$ of solvent five times for a total of $15 \mu \mathrm{L}$ directly onto the dried sample spot. This was immediately followed by pump B dispensing $10 \mu \mathrm{L}$ of solvent eight times for a total of $80 \mu \mathrm{L}$ at the rear of the cartridge. The spray solvent was $95 / 5$ methanol/water with $0.01 \%$ formic acid. MS analysis for the paper spray samples was performed using a Thermo Fisher Scientific Orbitrap Elite mass spectrometer. The temperature of the MS capillary inlet was set to $325^{\circ} \mathrm{C}$, and the tube lens voltage was set at $60 \mathrm{~V}$. The MS method run time was 1.0 minute, broken down into 4 time segments with varying spray voltages: $0-0.1 \mathrm{~min}, 0 \mathrm{kV} ; 0.1-0.8 \mathrm{~min},+5 \mathrm{kV} ; 0.8-0.9$ min, $0 \mathrm{kV}$; and 0.9-1.0 min, -5 kV. Tandem mass spectra were recorded using collisioninduced dissociation (CID). The precursor ions and primary fragment ions observed for the five simulant compounds were as follows: TMP $(\mathrm{m} / \mathrm{z} 141.0311 \rightarrow 127.0154)$ at CE $35 \mathrm{~V}, \mathrm{~d}_{9}$ TMP $(\mathrm{m} / \mathrm{z} 150.0877 \rightarrow 133.0531)$ at CE $35 \mathrm{~V}$, DMMP $(\mathrm{m} / \mathrm{z} 125.0362 \rightarrow$ $111.0205)$ at CE $32 \mathrm{~V}$, DIMP $(\mathrm{m} / \mathrm{z} 203.0807 \rightarrow 161.0338)$ at CE $30 \mathrm{~V}$, and ${ }^{13} \mathrm{Cd}_{3} \mathrm{DIMP}$ $(\mathrm{m} / \mathrm{z} 207.1029 \rightarrow 165.0560)$ at CE $30 \mathrm{~V}$.

Distribution Statement A: Approved for public release; distribution is unlimited. 
For $\mathrm{QqQ}$ samples, raw data was manually extracted and processed using MassHunter (Agilent Technologies). For PS samples run on the Orbitrap ELITE, raw data was manually extracted and processed using XCalibur 2.0 (Thermo Fisher Scientific). For samples run on each instrument, base peaks for each analyte transition were extracted from the total ion chromatogram (TIC), and the total area was determined. The analyte total area was used to determine the ratio relative to the total area of the fixed internal standard (ISTD). Each dilution of the standard curve was analyzed in technical triple. Calibration curves were generated by graphing averaged ratios with the known concentrations, and lines of best fit were calculated by linear regression. The limit of detection (LOD) was determined by multiplying the error in the $y$-intercept by 3.3 and dividing by the slope. Sample efficiency was calculated by the amount of aerosolized CWA simulant observed on substrate versus the total amount of simulant available for capture, which is then averaged across all concentrations for a general efficiency.

LODs which were calculated in $\mathrm{ng} / \mathrm{mL}$ were converted to $\mathrm{mg} / \mathrm{m}^{3}$ using Equation 1 below. Where LOD is the determined limit of detection, $F_{R}$ is the flow rate of the liquid to be aerosolized, $\mathrm{T}_{\mathrm{C}}$ is the time over which capture on the device occurred, and finally the $\mathrm{C}_{\mathrm{F}}$ which is the dilution air flow that the dispersed aerosol was sprayed into. It is important to note that this calculation is method dependent which considers capture time $\left(T_{C}\right)$ and, therefore, total volume of air sampled. Experimentally, $F_{R}$ was 0.01 $\mathrm{mL} / \mathrm{min}$ and $\mathrm{C}_{\mathrm{F}}$ was $20 \mathrm{~L} / \mathrm{min}$. Ultimately, this determines the quantity that is anticipated to be captured if the total volume sampled was an entire cubic meter. This relation is important for comparisons between LOD and permissible levels.

$$
\frac{\operatorname{LOD}\left(\frac{n g}{m L}\right) \times 1 \times 10^{-6}\left(\frac{m g}{n g}\right)}{\left[F_{R}\left(\frac{m L}{\min }\right) \times T_{C}(\min )\right]^{-1}} \div\left\{\left[C_{F}\left(\frac{L}{\min }\right) \times T_{C}(\min )\right] /\left[\frac{1000 L}{m^{3}}\right]\right\}=\text { Aerosol LOD } \frac{m g}{m^{3}}
$$

\section{Safety Considerations}

DMMP and DIMP are classified as Schedule 2 under the Chemical Weapons Convention $^{35}$, as they are precursors in CWA products. While suitable as simulants, there is need to be mindful of the retained toxicity of these compounds. Obey your organizations safety regulations and standard operating procedures while being mindful of national and international regulations regarding CWAs and associated simulants. Donning of appropriate protective equipment is advised.

\section{Results and Discussion}

Generation One:

Distribution Statement A: Approved for public release; distribution is unlimited. 
A holder for the PS cartridge was designed and 3D printed for collection directly onto the PS substrate, for rapid analysis of CWA simulants via PS MS. This device is shown in Figure 1 with the commercially available PS cartridge inside. The air intake aperture is positioned directly above and approximately $0.5 \mathrm{~cm}$ from the paper surface. Aerosolized CWA simulants were pumped from the chamber and drawn through the device inlet and over the paper substrate. This design was necessary to incorporate the commercially available disposable cartridge that was compatible with the Velox360 PS ion source.

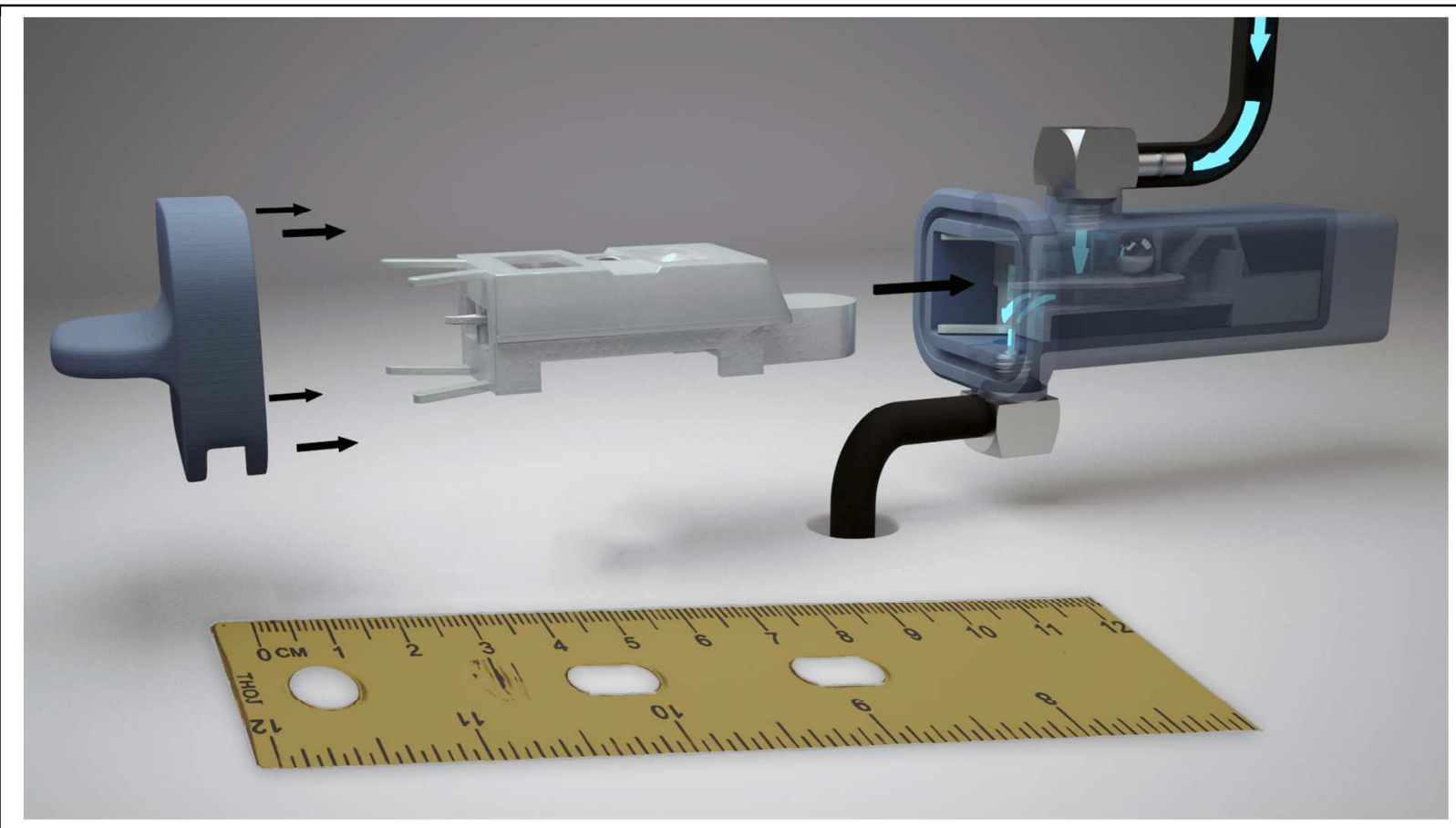

Figure 1. Generation one aerosol capture device with incorporation of the commercial PS cartridge. Airflow was directed toward the paper substrate before exiting the holder. Ruler shown for scale.

For comparison purposes, each concentration level aerosol was collected from the exposure chamber simultaneously in triplicate on the filter disk collection apparatus (Figure 2) and in quintuplicate on PS cartridges. The airflow through each of the collection devices was maintained at 1.5 liters per minute to assure the same volume of air sampled across each substrate. Figure 2 shows calibration curves of aerosolized CWA simulants collected on glass fiber disks followed by extraction and LC-QqQMS analysis (A) and collected directly on PS cartridges followed by Orbitrap MS analysis (B). Both sets of curves were linear with similar $R^{2}$ and LOD values in terms of disbursed aerosol concentration. Therefore, the PS method generated comparable results to the traditional filter disk extraction method, but PS analysis did not require the extraction step. This translates to faster sample preparation (few seconds vs. 60 minutes), less solvent usage, and faster analysis time (60 seconds vs. 10 minutes).

Distribution Statement A: Approved for public release; distribution is unlimited. 
While the glass fiber disk has a higher capture efficiency $(\approx 40 \%)$, the PS method produced undesirable results with a lower capture efficiency of $\sim 1-3 \%$ (Table 1).


Figure 2. Calibration curves of TMP, DMMP, and DIMP collected in the laboratory aerosol chamber for 10 minutes from traditional disk extracts analyzed on the $\mathrm{QqQ}$ (A) and paper spray cartridges (B).

\section{Table 1}

Distribution Statement A: Approved for public release; distribution is unlimited. 
Efficiency table of the first generation paper spray capture device.

\begin{tabular}{lccc}
\hline Generation 1 & DMMP & TMP & DIMP \\
\hline Filter Disk & $39.0 \%$ & $45.0 \%$ & $51.0 \%$ \\
Paper Spray & $0.7 \%$ & $1.4 \%$ & $3.3 \%$
\end{tabular}

The PS cartridge holder design was invited to be tested alongside many other chemical detectors at the US Army's S/K Challenge at Dugway Proving Grounds, Utah. At this event, CWA simulants were explosively disbursed, and several unmanned devices with a variety of sensors were flown (T-Hawk) or driven through the resulting cloud (Modular Detection and Response System, MDARS), as seen in Figure S1. The PS cartridge holder and pump (along with other detectors) were integrated into ACORNS (Array Configurable of Remote Network Sensors), a multi-sensor monitoring and collection platform shown in the inset of Figure S1. ACORNS was on the MDAR vehicle, while the PS holder was attached near an air intake port on the Honeywell THawk vehicle. During this field test, the only CWA simulant released that had been previously explored with PS in a laboratory environment was TMP.

Figure S1 also shows the results of each vehicle's run with the PS holder. The first two columns show the area of the TMP and the $d_{9}$ TMP from the extracted ion chromatogram, then the ratio of TMP to d9TMP was calculated. The amount of TMP on the PS cartridge was calculated using the PS-MS calibration curve of TMP standard (Figure S1). These numbers were used to calculate the average concentration of TMP collected during the flight through the aerosol cloud. The concentrations in the cloud were likely up to a factor of two higher than this because the vehicles were not in the aerosol cloud for the entire duration of their flight time, but the pumps were on for the whole flight. Unfortunately, the volume of air sampled by the T-Hawk vehicle is unknown due to an atypical deployment, so it was not possible to calculate a minimum amount of TMP in the aerosol cloud during that run.

\section{Generation Two}

Due to the gross difference in capture efficiency between the generation one PS holder and the traditional aerosol disk extraction method, a redesign of the holder and capture substrate was necessary. A comparison of substrates using the Whatman Chromatography ET31 paper in the aerosol capture holder (Figure S2) demonstrated improved area counts relative to the glass fiber filter by $\mathrm{QqQ}$ analysis. Therefore, it was determined that the aerosol needed to be drawn through the substrate. The back of the PS cartridge was milled away to expose the underside of the Whatman paper (Figure S3), and raised islands were added to the second generation holder, as seen in Figure 3. The paper substrate was mated between the two raised islands. However, preliminary tests indicated that the Whatman paper in the commercial PS cartridges

Distribution Statement A: Approved for public release; distribution is unlimited. 
was not chemically and physically robust enough to be a viable substrate during this application.

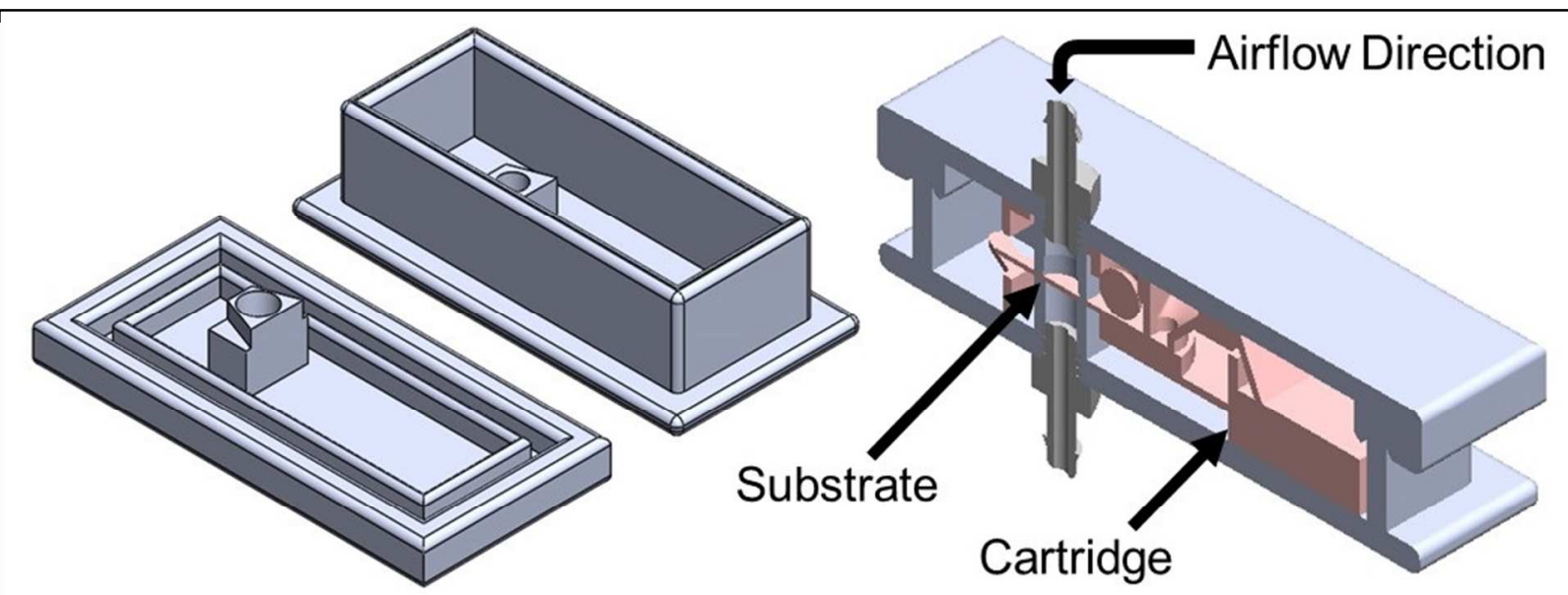

Figure 3. Generation two design. Raised islands were added which were designed to mate flush with the paper spray cartridge to force airflow through the new substrate.

The glass fiber filter substrate used in the filter aerosol collection was explored as a replacement PS substrate. Figure 4A shows the average peak areas for the three CWA simulants when analyzed by Whatman paper, untreated glass fiber filter, and the glass fiber filter treated with $500 \mathrm{mM}$ ammonium sulfate. When untreated glass fiber filters were used, the peak area counts for DIMP increased by approximately two orders of magnitude. However, protonated TMP and DMMP were not seen in the spectra. This is likely due to the fact that TMP and DMMP more readily interact with hydroxylated surfaces ${ }^{39-40}$. While, DIMP is the most polar of the simulants and is still available to spray ${ }^{41}$. The filtration disks were treated with ammonium sulfate in an effort to deactivate the glass surface. As a result, the ionizations of TMP and DMMP were highly increased, and the peak areas for all three CWA simulants were increased when compared to the Whatman paper substrate.

Figure 4B shows the PS calibration curves using the treated glass substrate. All three CWA simulants were linear with high $R^{2}$ values. The LOD values for all three simulants were comparable to LODs calculated using the commercially available PS cartridges (Figure S2B). Therefore, both substrates were comparable for the CWA simulants.

Distribution Statement A: Approved for public release; distribution is unlimited. 

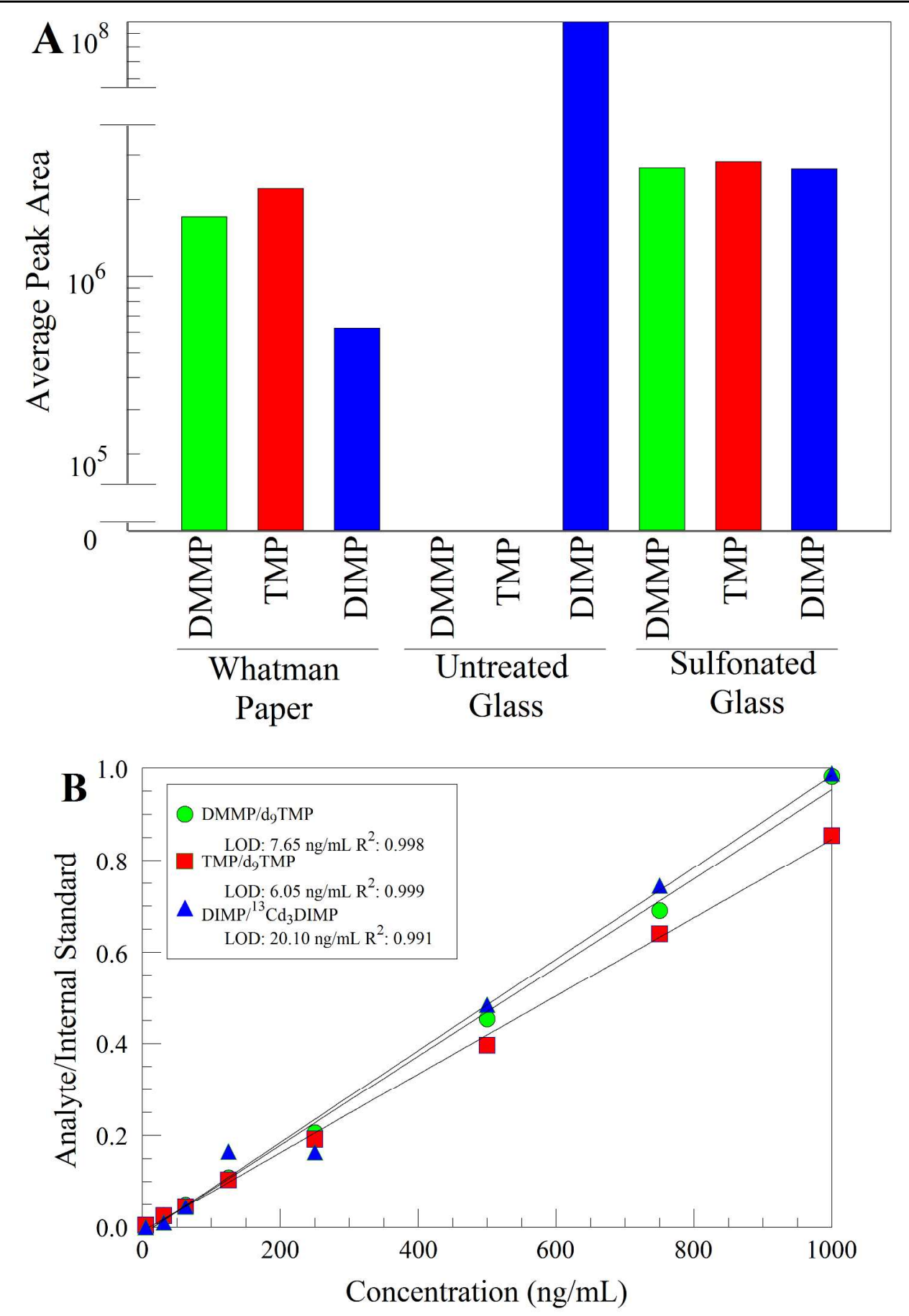

Figure 4. (A) Tabulation of peak areas obtained from $1,000 \mathrm{ng} / \mathrm{mL}$ concentrations of DMMP, TMP, and DIMP sprayed off various substrates to indicate the effectiveness of surface chemistry changes of the substrate. (B) Linear calibration curve from sulfonated glass substrate used for paper spray, reconfirming the effectiveness of the improved substrate material.

Distribution Statement A: Approved for public release; distribution is unlimited. 
The generation two holder and sulfonated glass fiber substrate were tested in an aerosol chamber against treated filter capture apparatus. Two iterations of calibration standards were aerosolized, and each calibration level was collected in triplicate on treated glass fiber filtration disks and in triplicate on treated glass PS cartridges. Figure S5 shows the high (3-180 ppm) iteration of the calibration curve, and Figure 5 shows the low $(2-500 \mathrm{ppb})$ iteration of the calibration curve. The aerosol was sampled for a reduced time of 2 minutes in an effort to reduce collection time and lower the amount collected on substrate. All three CWA simulant curves had reasonable $\mathrm{R}^{2}$ values for aerosol collection. The linear dependence of the curves stopped at $\approx 50 \mathrm{ppb}$, but signal was detectable to $5 \mathrm{ppb}$. However, traditional LOD calculations were done in an effort to be conservative regarding the performance of the second generation PS holder, and the simulant LOD values were calculated in the low ppb-range.

These LOD numbers are within worker population limits ${ }^{42}$ of warfare agent exposures. Moreover, detection and subsequent analysis occurred faster (minutes vs. hours) than the current state-of-the-art methods ${ }^{43}$. Additionally, the change in substrate resulted in comparable capture efficiencies between the filter capture-extraction and PS capture, as seen in Table 2. This increased efficiency ultimately provides more available analyte for subsequent analysis post capture, driving down the LOD. Relating these capture results to personnel exposure limits, Table 2 compares the determined LOD from a 2 minute capture utilizing Equation 1 and the determined limit of exposure. These LODs can be compared to the Worker Population Limit (WPL) which is described as the exposure allowed for 8 hours per day during the work week, with no observable effects. By increasing capture time, it may be possible to detect General Population Limits (GPL), which are the maximum concentrations allowed for continuous 24 hours a day, indefinite exposure with no observable effects.

Distribution Statement A: Approved for public release; distribution is unlimited. 

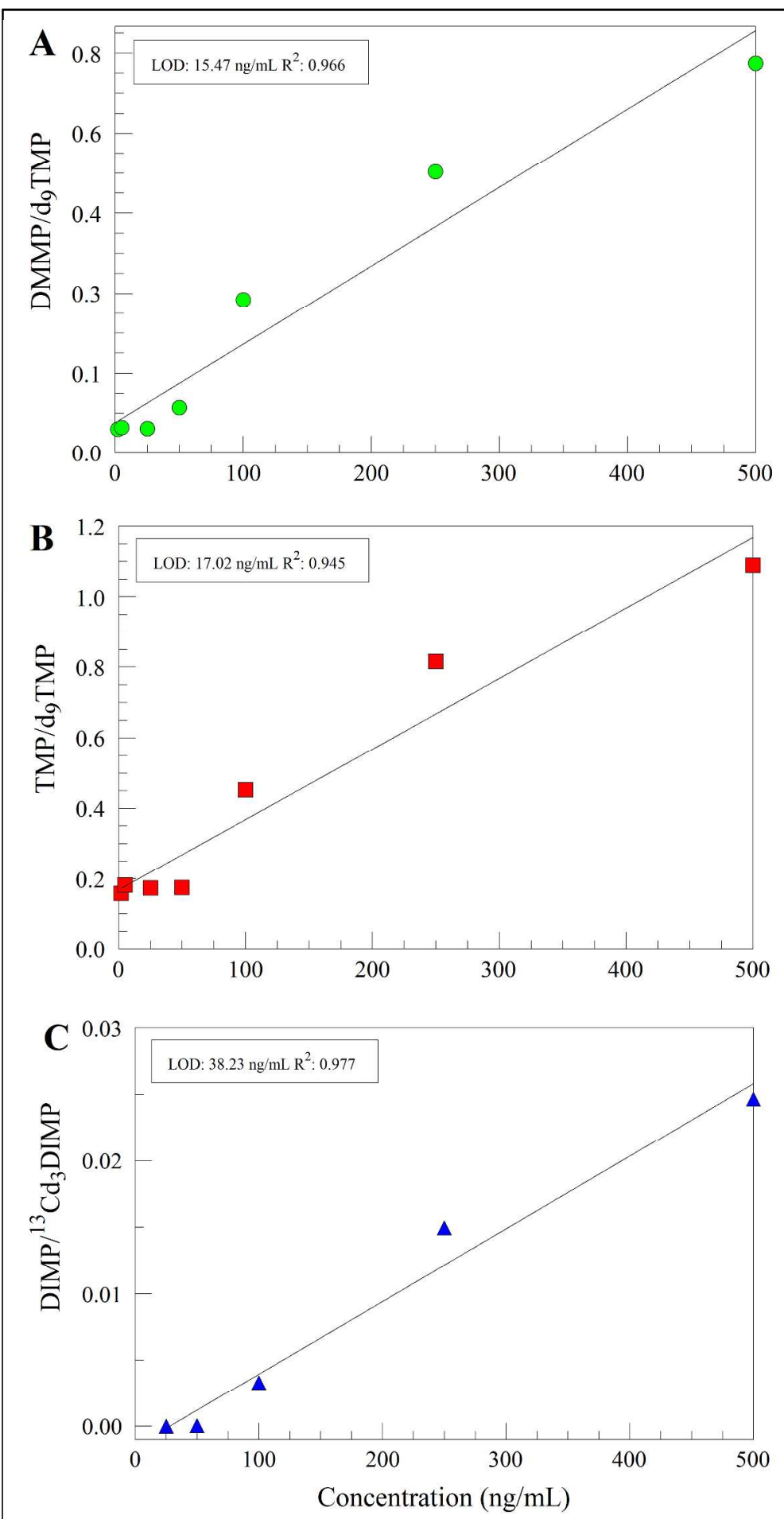

Figure 5. Obtained aerosol capture curves for 2 minutes at ppb levels of the DMMP (A), TMP (B), and DIMP (C). For DIMP, lowest two concentrations excluded due to nonresponse.

Distribution Statement A: Approved for public release; distribution is unlimited. 


\section{Table 2}

Efficiency tables of the final generation of paper spray capture device, with flow through sulfonated glass fiber substrate, compared to traditional glass fiber aerosol filtration disk. Comparison of tabulated limits of detection in the final improved generation compared to established permissible levels established by the U.S. Army Public Health Command ${ }^{40}$.

\begin{tabular}{lccc}
\hline Generation 2 & DMMP & TMP & DIMP \\
\hline Filter Disk & $50.0 \%$ & $38.6 \%$ & $48.5 \%$ \\
Paper Spray & $52.9 \%$ & $49.3 \%$ & $44.9 \%$ \\
\hline
\end{tabular}

\begin{tabular}{ccc|cc|cc}
\multicolumn{2}{c|}{$\begin{array}{c}\text { PS Aerosol LOD } \\
\left(\mathrm{mg} / \mathrm{m}^{3}\right)\end{array}$} & \multicolumn{2}{c|}{$\begin{array}{c}\text { Worker Population Limit } \\
\left(\mathrm{mg} / \mathrm{m}^{3}\right)\end{array}$} & \multicolumn{2}{c}{$\begin{array}{c}\text { General Population } \\
\text { Limit }\left(\mathrm{mg} / \mathrm{m}^{3}\right)\end{array}$} \\
DMMP & TMP & DIMP & G-Series & VX & G-Series & VX \\
$7.74 \times 10^{-6}$ & $8.51 \times 10^{-6}$ & $1.91 \times 10^{-5}$ & $3.0 \times 10^{-5}$ & $1.0 \times 10^{-6}$ & $1.0 \times 10^{-6}$ & $6.0 \times 10^{-7}$ \\
\hline
\end{tabular}

\section{Conclusions}

PS cartridges were able to capture aerosolized CWA simulants using a 3D printed holder. The analysis of these simulants demonstrated that PS-MS produces comparable results to the method of filter collection, extraction, and analysis by $\mathrm{QqQ}$ LC-MS. The PS method does not require an extraction step, which allows for simple sample preparation, less solvent use, and faster analysis time. The PS cartridge holder was also successfully integrated into unmanned aerial and ground vehicles for realworld testing, which generated positive results in the ability to confidently capture and identify CWA simulants down to tens of nanogram levels, as well as approximate the concentration of aerosolized simulant in the cloud. This technique for aerosol capture and analysis is certainly amendable to in-field analysis of actual CWA compounds. There are now many fielded portable systems compatible with ambient ionization techniques, including Direct Analysis in Real Time (DART) ${ }^{44}$ PS $^{45}$, and Desorption Electrospray lonization $(\mathrm{DESI})^{45}$. This would aid in higher throughput, and reduced contamination from CWAs in analytical laboratories.

Further iterative holder designs included bringing the proximity of the inlet to the substrate surface and increasing the volume of air sampled per unit time. Glass fiber filters were investigated and determined to be a suitable capture and spray substrate. The unmodified glass's reactivity with the simulants resulted in less than desirable performance, as the simulants interacted with the glass. However, sulfonating the glass fiber filters allowed for equal capture and analysis of all the choosen simulants, removing any glass substrate effects that caused loss of signal. Low $\mathrm{mg} / \mathrm{m}^{3}$ LODs were achieved for the three CWA stimulants, which are comparable to the WPL set out by

Distribution Statement A: Approved for public release; distribution is unlimited. 
U.S. Army Public Health Command. Anticipated further improvements in the substrate would allow for detection of general population limits ${ }^{42}$ of CWAs.

\section{Acknowledgements}

The authors would like to thank Jason Gitlin, Lester Strauch, III, Dr. Alan Samuels and for their support in completing this research. The authors would also like to especially recognize Dr. Jennifer Sekowski, Dr. Bao Q.Tran, Dr. Michael Feasel, and David McCaskey for productive conversations and Dr. Robert Bernhards for review of the manuscript. A special thanks to Dr. Bob Williams and Mark Alverez at Los Alamos National Lab for the synthesis of ${ }^{13} \mathrm{Cd}_{3} \mathrm{DIMP}$. This research was performed while the author (E.S.D.) held a NRC Research Associateship Award at Edgewood Chemical Biological Center. Thanks to Advanced Design and Manufacturing Division at E.C.B.C and Dugway Proving Grounds for their infrastructure and support. The authors would like to thank Donna Hollinshead and Dr. Justin Wiseman at Prosolia for the use of the Velox360 PS source.

Funding for this project was provided by the Defense Threat Reduction Agency-Joint Science and Technology Office for Chemical and Biological Defense to T.G. Conclusions and opinions presented here are those of the authors and are not the official policy of the U.S. Army, ECBC, or the U.S. Government. Information in this report is cleared for public release and distribution is unlimited.

Supporting Information Available: Field Trials, Drawings of Capture Devices, Modified Products, and additional datasets.

\section{References}

1. Geneva Protocol. Government of France, Protocol for the prohibition of the use in war of asphyxiating, poisonous or other gases, and of bacteriological methods of warfare. U.S. Department of State: Bureau of International Security and Nonproliferation. Signed at Geneva, June 17. 1925

2. Kellman, B., J. Legis. 1999, 25, 117.

3. Pita, R.; Domingo, J., Toxics 2014, 2, 391-402.

4. Zarocostas, J., The Lancet 2017, 389, 1501.

5. Hill, H. H.; Martin, S. J., Pure Appl. Chem. 2002, 74, 2281-2291.

6. Sferopoulos, R. A Review of Chemical Warfare Agent (CWA) Detector Technologies and Commercial-Off-The-Shelf Items; Austrailian Government March 2009, 2009.

7. Hill, H. H.; Simpson, G., Field Anal. Chem. Technol. 1997, 1, 119-134.

8. Steiner, W. E.; Klopsch, S. J.; English, W. A.; Clowers, B. H.; Hill, H. H., Anal. Chem. 2005, 77, 4792-4799.

9. Pacsial-Ong, E. J.; Aguilar, Z. P., Front. Biosci. 2013, 5, 516-543.

Distribution Statement A: Approved for public release; distribution is unlimited. 
10. Giannoukos, S.; Brkić, B.; Taylor, S.; France, N., J. Am. Soc. Mass. Spectrom. 2015, 26, 231-239.

11. Mach, P. M.; McBride, E. M.; Sasiene, Z. J.; Brigance, K. R.; Kennard, S. K.; Wright, K. C.; Verbeck, G. F., Anal. Chem. 2015, 87, 11501-11508.

12. Sun, Y.; Ong, K. Y., Detection technologies for chemical warfare agents and toxic vapors. 1st ed.; CRC Press: Boca Raton, FL, 2005.

13. Longworth, T. L.; Ong, K. Y. Domestic Preparedness Program Testing of the CAM-Chemical Agent Monitor (Type L) Against Chemical Warfare Agents Summary Report; DTIC Document: 2002.

14. Ong, K. Y.; Longworth, T.; Barnhouse, J. L. Domestic Preparedness Program: testing of APD2000 Chemical Warfare Agent Detector against chemical warfare agents summary report; DTIC Document: 2001.

15. Derringer, T.; Kelly, T.; Folsom, D.; Krile, R.; Willenberg, Z.; Koglin, E. Technology Evaluation Report: Environics USA Inc. ChemPro 100 Hand-Held Chemical Detector, Battelle, Columbus, OH, 2006; pp 1-49.

16. Kosal, M. E., Center for Nonproliferation Studies: USA 2003.

17. Mach, P. M.; Wright, K. C.; Verbeck, G. F., J. Am. Soc. Mass Spectrom. 2015, 26, 281-285.

18. Srinivasan, N.; Johnson, R.; Kasthurikrishnan, N.; Wong, P.; Cooks, R., Anal. Chim. Acta 1997, 350, 257-271.

19. Ketola, R.; Ojala, M.; Sorsa, H.; Kotiaho, T.; Kostiainen, R., Anal. Chim. Acta 1997, 349, 359-365.

20. DeCarlo, P. F.; Kimmel, J. R.; Trimborn, A.; Northway, M. J.; Jayne, J. T.; Aiken, A. C.; Gonin, M.; Fuhrer, K.; Horvath, T.; Docherty, K. S., Anal. Chem. 2006, 78, 82818289 .

21. Jayne, J. T.; Leard, D. C.; Zhang, X.; Davidovits, P.; Smith, K. A.; Kolb, C. E.; Worsnop, D. R., Aerosol Sci. Technol 2000, 33, 49-70.

22. Kanawati, B.; Herrmann, F.; Joniec, S.; Winterhalter, R.; Moortgat, G. K., Rapid Commun. Mass Spectrom. 2007, 22, 165-186.

23. McMurry, P. H., Atmos. Environ. 2000, 34, 1959-1999.

24. Suess, D. T.; Prather, K. A., Chem. Rev. 1999, 99, 3007-3036.

25. Martin, A. N.; Farquar, G. R.; Frank, M.; Gard, E. E.; Fergenson, D. P., Anal. Chem. 2007, 79, 6368-6375.

26. Zelenyuk, A.; Imre, D.; Wilson, J.; Zhang, Z.; Wang, J.; Mueller, K., J. Am. Soc. Mass. Spectrom. 2015, 26, 257-270.

27. Liu, J.; Wang, H.; Manicke, N. E.; Lin, J.-M.; Cooks, R. G.; Ouyang, Z., Anal. Chem. 2010, 82, 2463-2471.

28. Zhang, C.; Manicke, N. E., Analytical chemistry 2015, 87, 6212-6219.

29. Kerian, K. S.; Jarmusch, A. K.; Cooks, R. G., Analyst 2014, 139, 2714-2720.

30. Wang, H.; Manicke, N. E.; Yang, Q.; Zheng, L.; Shi, R.; Cooks, R. G.; Ouyang, Z., Anal. Chem. 2011, 83, 1197-1201.

31. Wang, H.; Liu, J.; Cooks, R. G.; Ouyang, Z., Angew. Chem. Int. Ed. 2010, 122, 889-892.

32. Evard, H.; Kruve, A.; Lõhmus, R.; Leito, I., J. Food Compos. Anal. 2015, 41, $221-$ 225.

Distribution Statement A: Approved for public release; distribution is unlimited. 
33. McKenna, J.; Dhummakupt, E. S.; Connell, T.; Demond, P.; Miller, D. B.; Nilles, J. M.; Manicke, N.; Glaros, T., Analyst 2017.

34. Reeber, S. L.; Gadi, S.; Huang, S.-B.; Glish, G. L., Anal. Meth. 2015, 7, 98089816.

35. Bartelt-Hunt, S. L.; Knappe, D. R.; Barlaz, M. A., Crit. Rev. Env. Sci. Technol. 2008, 38, 112-136.

36. Black, R. M.; Muir, B., J. Chromatogr. A 2003, 1000, 253-281.

37. Yashchishin, I.; Zheplinskii, T., Glass Ceram. 1996, 53, 135-137.

38. McCaskey, D. A. Coaxial needle atomizing system. US 9016671 B1, 2015.

39. Davis, E. D.; Gordon, W. O.; Wilmsmeyer, A. R.; Troya, D.; Morris, J. R., J. Phys. Chem. Lett. 2014, 5, 1393-1399.

40. Kanan, S. M.; Tripp, C. P., Langmuir 2001, 17, 2213-2218.

41. Wilmsmeyer, A. R.; Gordon, W. O.; Davis, E. D.; Troya, D.; Mantooth, B. A.; Lalain, T. A.; Morris, J. R., J. Phys. Chem. C 2013, 117, 15685-15697.

42. Preventive Medicine Data: AR 40-5e PHN No. 0711-02. Standards and Guidelines Summary; Table 1: Criteria for Airborne Exposures. U.S. Army Public Health Command. 2011

43. Harper, M., J. Environ. Monit. 2002, 4, 688-694.

44. Brown, H.; Oktem, B.; Windom, A.; Doroshenko, V.; Evans-Nguyen, K., For. Chem. 2016, 1, 66-73.

45. Lawton, Z. E.; Traub, A.; Fatigante, W. L.; Mancias, J.; O'Leary, A. E.; Hall, S. E.; Wieland, J. R.; Oberacher, H.; Gizzi, M. C.; Mulligan, C. C., J. Am. Soc. Mass Spectr. 2016, 1-12.

Distribution Statement A: Approved for public release; distribution is unlimited. 


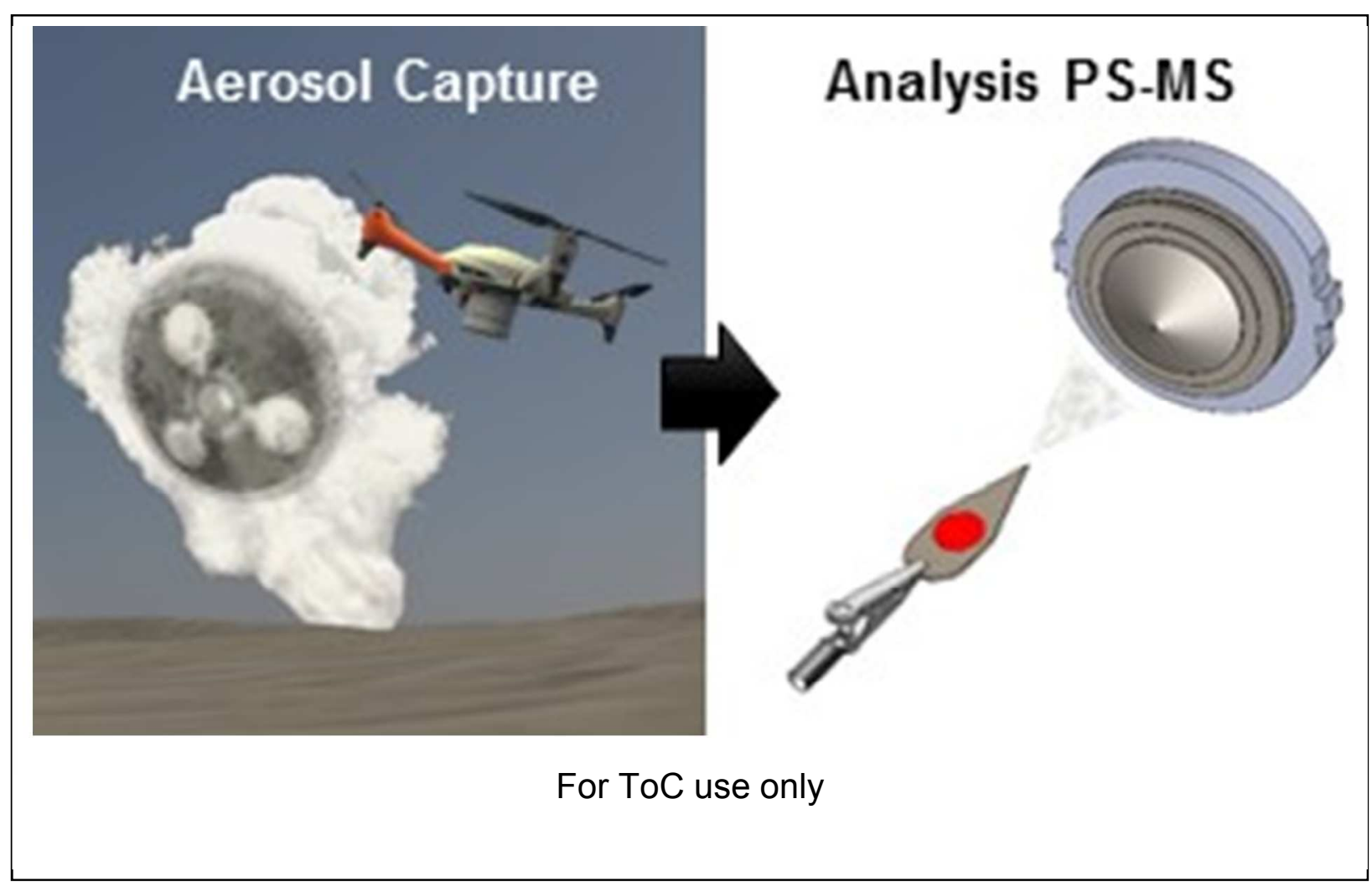

Distribution Statement A: Approved for public release; distribution is unlimited. 


\section{Supporting Information For:}

\section{Direct Analysis of Aerosolized Chemical Warfare Simulants Captured on a Modified Glass-Based Substrate by ‘Paper-Spray’ Ionization}

Elizabeth S. Dhummakupt ${ }^{\dagger,}$, Phillip M. Mach ${ }^{\ddagger}$, , Daniel Carmany ${ }^{\ddagger}$, Paul S. Demond ${ }^{\ddagger}$, Theodore S. Moran ${ }^{\dagger}$, Theresa Connell ${ }^{\ddagger}$, Harold S. Wylie", Nicholas E. Manicke ${ }^{\perp}, J$. Michael Nilles ${ }^{\ddagger, *}$, and Trevor Glaros ${ }^{\dagger, *}$

† Research and Technology Directorate, US Army Edgewood Chemical Biological Center (ECBC), Aberdeen Proving Ground, MD 21010, USA

‡ Excet, Inc. 6225 Brandon Ave, Suite 360, Springfield, VA 22150, USA

" TriMech Services, LLC, 4461 Cox Rd \# 302, Glen Allen, VA 23060, USA

${ }^{\perp}$ Department of Chemistry and Chemical Biology, Indiana University-Purdue University Indianapolis, Indianapolis, IN 46202, USA

Corresponding Authors

*T.G. trevor.g.glaros.civ@mail.mil

*J.M.N. mnilles@gmail.com

Table of Contents

S-2 S/K Challenge Field Trials Figure and Data

S-3 Comparison of Triple Quadrupole versus PS-MS Datasets

S-5 Figure of Traditional Aerosol Capture Device

S-6 Modified commercial PS Cartridge

S-7 Higher level concentration curve. 


\section{Supplemental Figures}

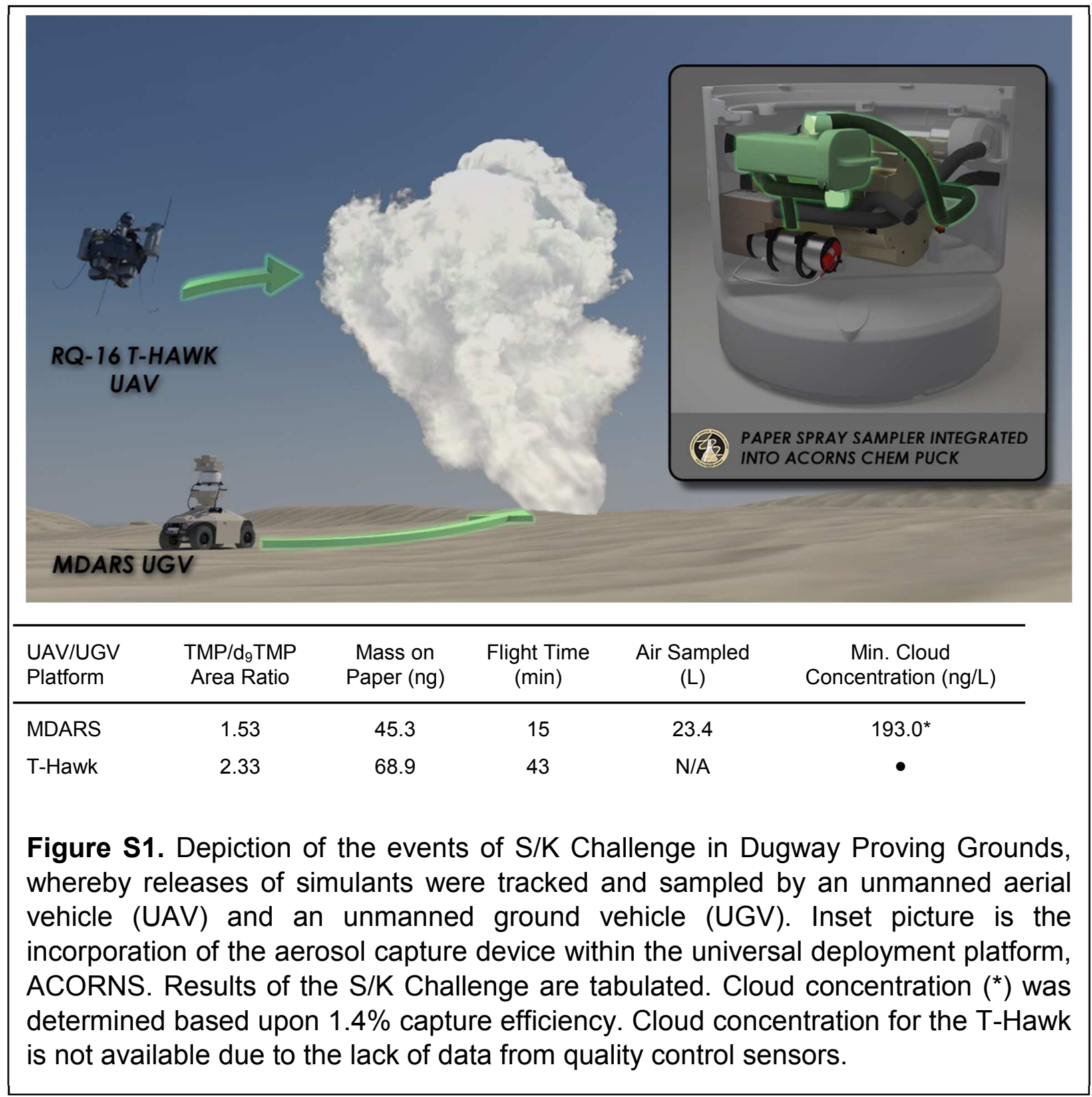


Calibration curves for TMP, DIMP, and DMMP relative to isotopically labeled internal standards were prepared using three different mass spectrometric methods. The curves for these simulants are illustrated in Figure S2. The primary method of aerosol quantitative analysis is collection on filter disks followed by liquid extraction and analysis on a triple quadrupole MS. Therefore, the calibration curve in Figure S2A is generated by direct application of analyte to fiber filters followed by extraction, addition of internal standard, and LC-QqQMS analysis. For comparison, PS-MS analysis was performed using the calibration solutions from the disk extraction and LC-QqQMS method. While all three techniques are linear and produce $R^{2}$ values near 1 , the current method of quantitative aerosol analysis (Figure S2B) produces larger limits of detection (LOD) and poorer $\mathrm{R}^{2}$ values due to extensive extraction and sample handling. The lack of sample handling for analysis by PS-MS (Figure 2C) contributes to improved $R^{2}$ and LOD values compared to the current method of aerosol analysis. Therefore, PS-MS is comparable to the direct analysis on the $\mathrm{QqQ}$. 

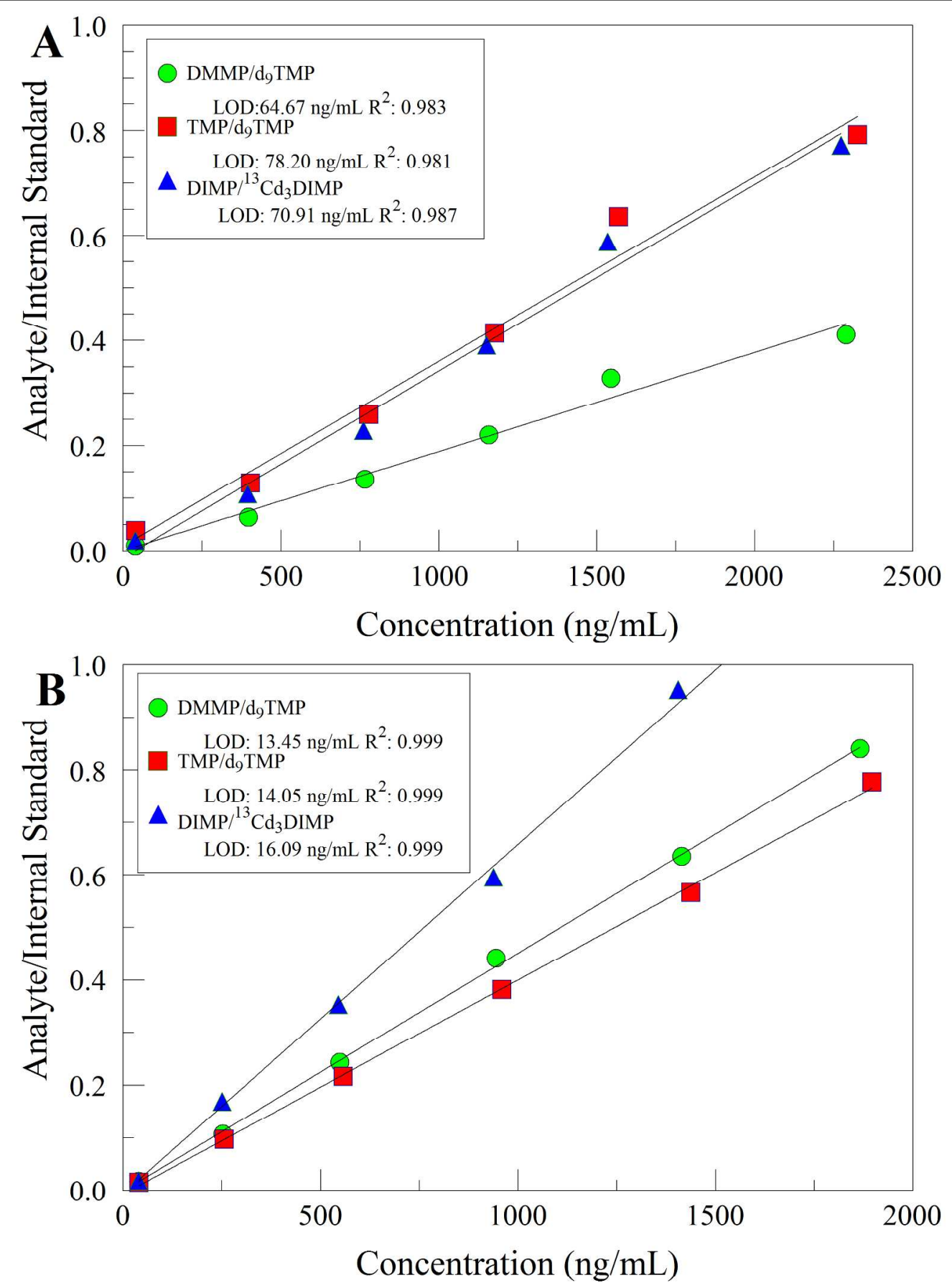

Figure S2. Calibration curves of DMMP, TMP, and DIMP standards by two different methods with corresponding LOD and $R^{2}$ values: extraction of standards from aerosol disk and analyzed via QqQ (A), and DMMP, TMP, and DIMP standards spotted on cartridge and analyzed via PS-MS (B). 


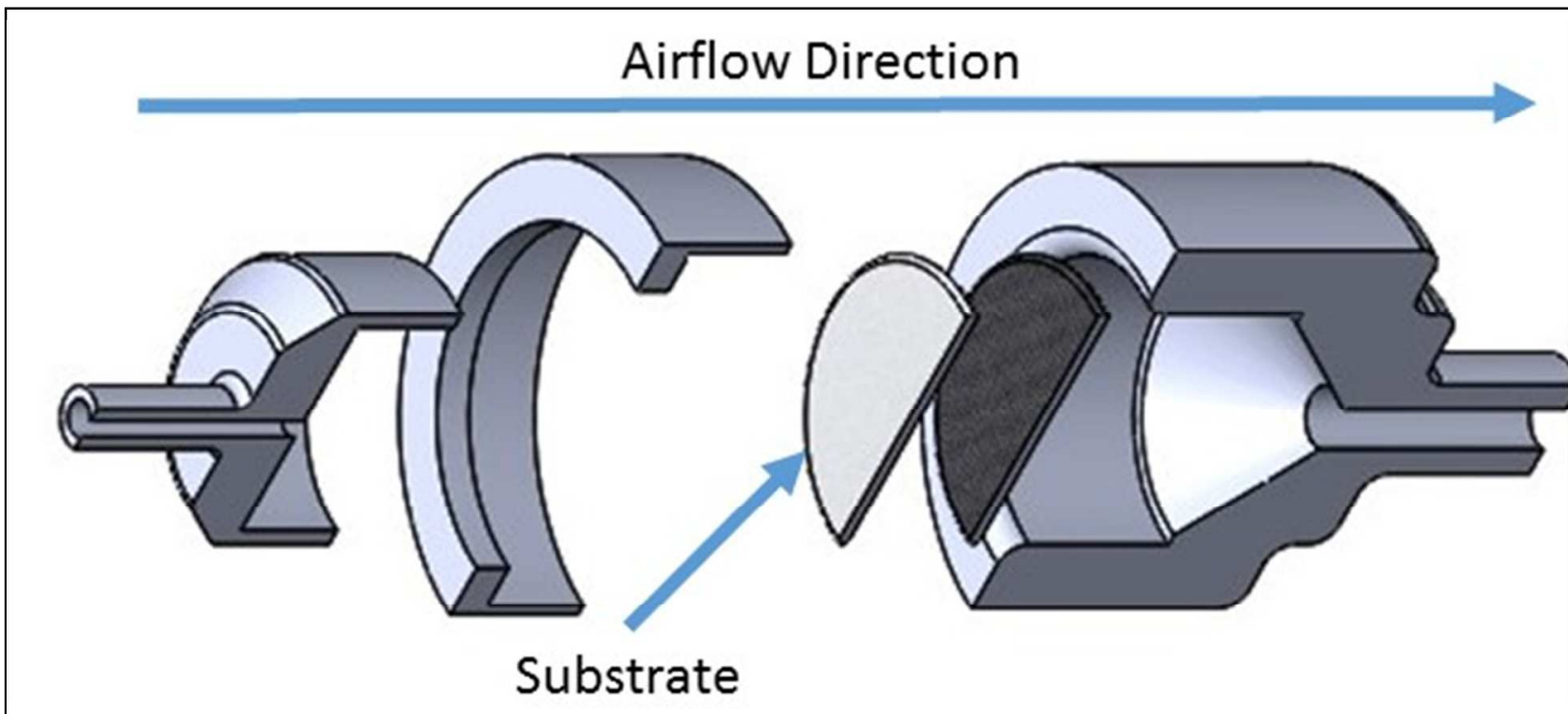

Figure S3. Traditional aerosol capture device where a substrate disk is placed within a sample stream. 


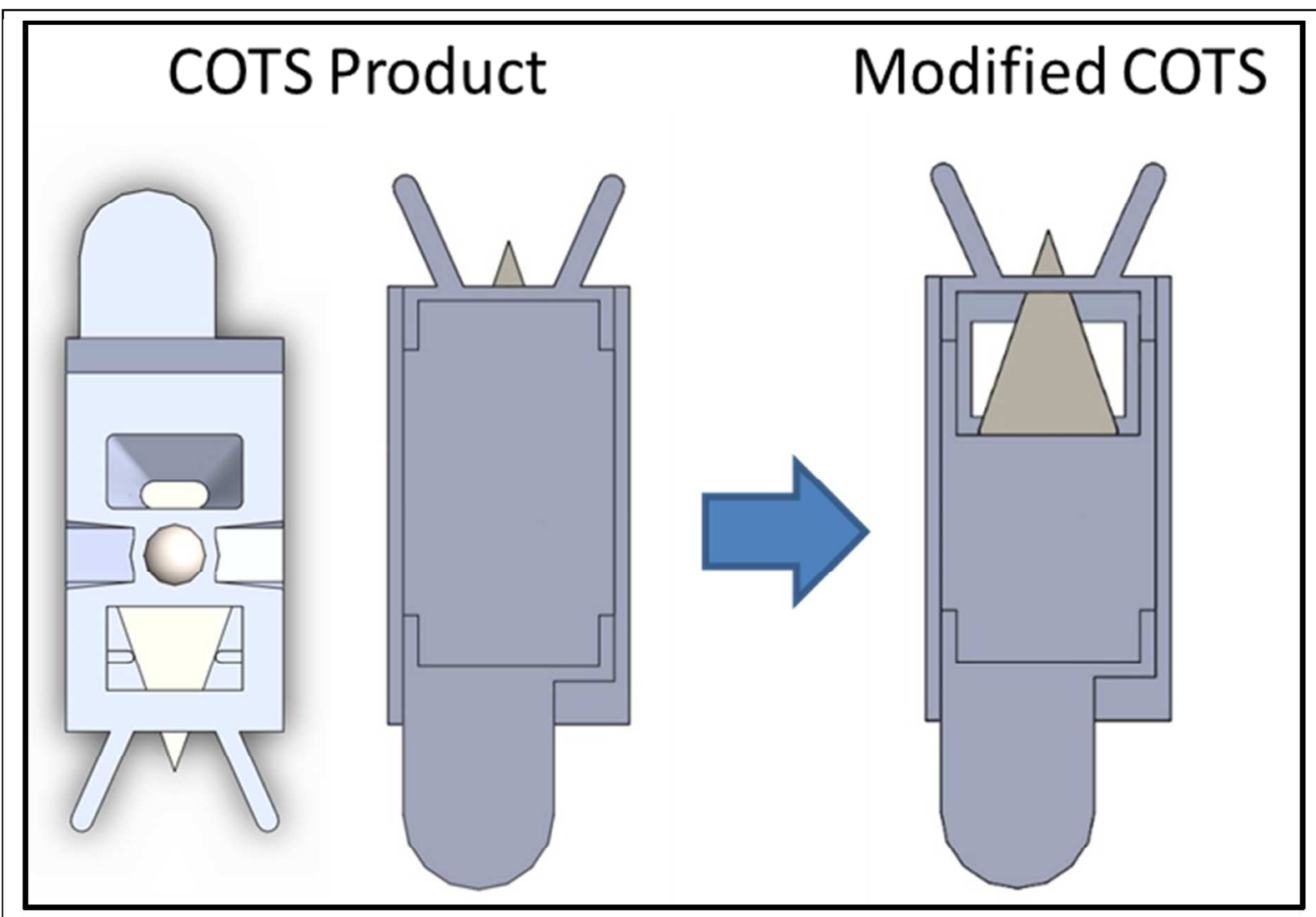

Figure S4. Modified commercial off the shelf (COTS) paper spray cartridge to allow for airflow through the substrate. 


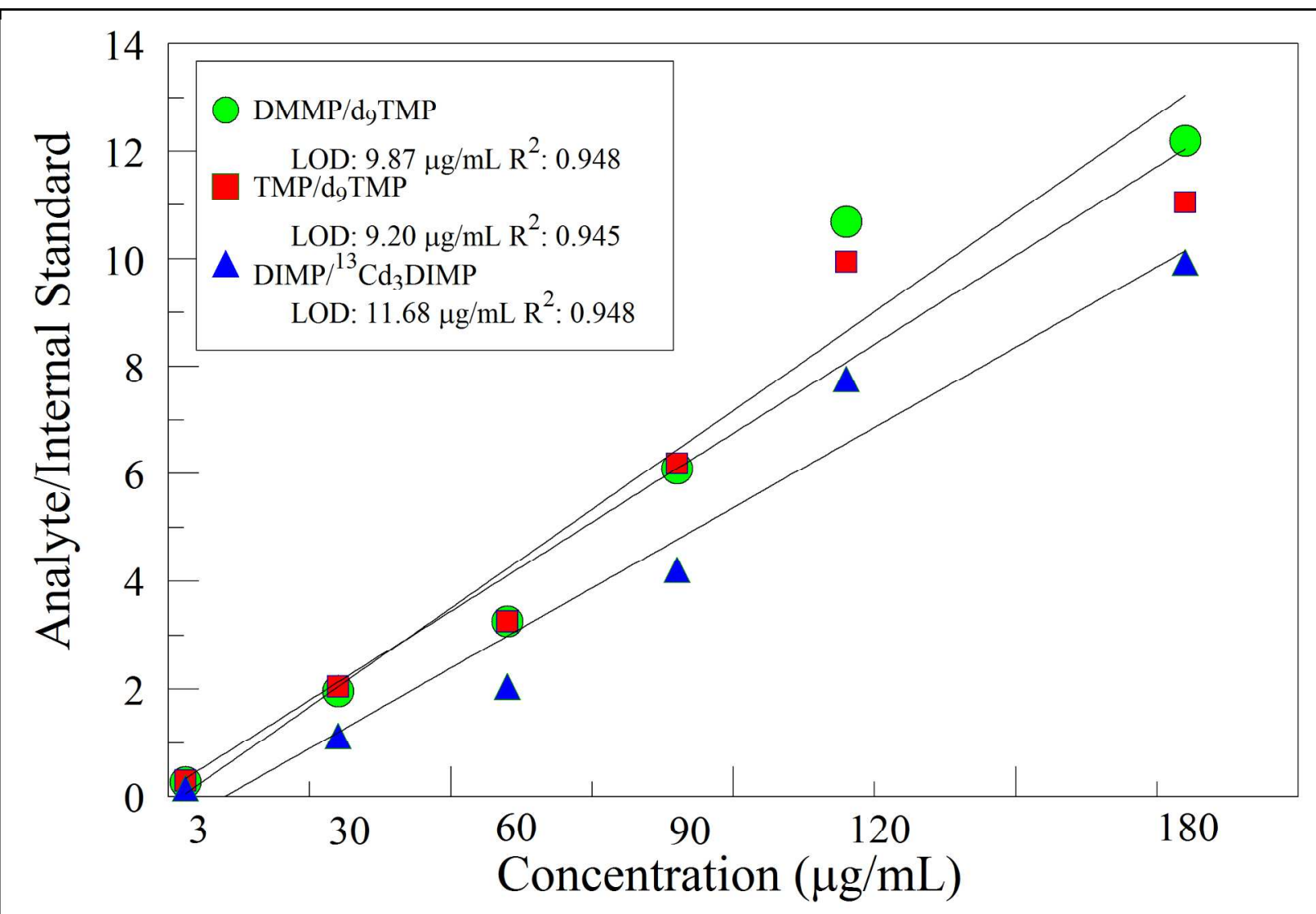

Figure S5. Treated glass substrate aerosol collection curve at higher concentrations. 